\title{
Relationship between body fat and diabetic retinopathy in patients with type 2 diabetes: a nationwide survey in Korea
}

\author{
In Cheol Hwang ${ }^{1} \cdot$ Jeong Hun $\mathrm{Bae}^{2} \cdot$ Joon Mo Kim²
}

Received: 19 January 2018 / Revised: 9 November 2018 / Accepted: 16 November 2018 / Published online: 13 February 2019

(c) The Royal College of Ophthalmologists 2019

\begin{abstract}
Purpose To evaluate the association of body mass index (BMI), waist circumference, and body fat with diabetic retinopathy (DR) in Korean patients with type 2 diabetes.

Methods Among the 1130 participants 19 years of age or older with type 2 diabetes enrolled in the nationwide survey, a total of 887 participants were included in the analyses after excluding patients with ungradable fundus images. The presence and severity of DR were graded with seven-field fundus photographs. The associations between DR and anthropometric parameters and total body fat, which was assessed by dual-energy X-ray absorptiometry, were analysed using multivariate logistic regression models.

Results Patients were divided into three groups according to severity of DR: no DR $(n=702,79.1 \%)$, mild/moderate DR $(n=130,14.7 \%)$, and vision-threatening DR $(n=55,6.2 \%)$. In multivariate-adjusted analyses, higher BMI (adjusted odds ratio $[\mathrm{aOR}], 0.80 ; 95 \%$ confidence interval $[\mathrm{CI}], 0.70-0.92 ; P=0.001)$, larger waist circumference $(\mathrm{aOR}, 0.96 ; 95 \% \mathrm{CI}$, $0.91-1.00 ; P=0.047$ ), and higher total body fat (aOR, $0.86 ; 95 \% \mathrm{CI}, 0.79-0.92 ; P<0.001$ ) were significantly associated with a lower risk of vision-threatening DR. In sex-stratified analyses, however, only total body fat in women showed a significant inverse association with the presence of DR (aOR, 0.93; 95\% CI, 0.88-0.98; $P<0.001)$ and independently with the severity of DR $(P$ for trend $=0.004)$.

Conclusion In Korean patients with type 2 diabetes, obese patients might be less likely to have vision-threatening DR. Total body fat in women appears to be inversely and independently associated with DR prevalence and progression.
\end{abstract}

\section{Introduction}

The prevalence of diabetes has rapidly increased worldwide, and 592 million people are expected to have diagnosed diabetes by 2035 [1]. The global epidemic of diabetes will possibly give rise to an increase in diabetic complications including diabetic retinopathy (DR), which is the most common microvascular complication in diabetic patients [2]. Diabetic retinopathy is a leading cause of blindness in working-age people, with vision-threatening complications

Jeong Hun Bae

jhbae94@hotmail.com

1 Department of Family Medicine, Gil Medical Center, Gachon University College of Medicine, Incheon, Republic of Korea

2 Department of Ophthalmology, Kangbuk Samsung Hospital, Sungkyunkwan University School of Medicine, Seoul, Republic of Korea in up to $10 \%$ of diabetic patients [3, 4]. Although previous epidemiologic studies have demonstrated that poor glycemic control, hypertension, dyslipidemia, and a longer duration of diabetes increase the risk of DR, the known effects of controlling glucose and blood pressure are limited in reducing the risk of DR [5-9]. Thus, other pathogenic or risk factors are important in understanding the development or progression of DR.

Body mass index (BMI) is a popular indicator of obesity, which is considered a risk factor for type 2 diabetes [10]. Higher BMI is presumably a risk factor in diabetic patients for cardiovascular morbidity or mortality and nephropathy [11-13]. However, results from previous studies that investigated the relationship between BMI and DR have been contradictory. Some studies have suggested that obesity or higher BMI increase the risk of DR [6, 14-16], but other studies have reported no association or even conflicting results in which higher BMI is associated with lower prevalence of DR [17-20]. The discordance in the association between BMI and DR might in part be due to the 
limitation of BMI as a measure of obesity. Emerging evidence indicates that measuring central or abdominal obesity, such as waist circumference or waist-hip ratio, is a better anthropometric marker of obesity-related metabolic risk than BMI $[6,18,21]$. In fact, the human body is composed of various tissues such as fat, muscle, bone, and water, but BMI is calculated using only body weight and height, with a score of 30 or more in Caucasians and 25 or more in Asians regarded as obesity. Since BMI does not provide information on adiposity, evaluating the effects of specific bodily traits on DR would be more appropriate.

Dual-energy X-ray absorptiometry (DXA) is a widely used method for measuring body composition such as bone mineral and soft tissue, which includes fat, lean body mass, and appendicular skeletal muscle [22]. In this study, we used data from a nationwide cross-sectional cohort of the Korean population to investigate the association of DR with anthropometric parameters of obesity and total body fat as assessed by DXA.

\section{Subjects and methods}

\section{Data source and study participants}

This study was based on data obtained from the Korean National Health and Nutrition Examination Survey (KNHANES) 2008-2010, which is a nationwide populationbased survey conducted periodically by the Korean Ministry of Health and Welfare. Participants were randomly selected through a stratified, multistage, probability-sampling design according to sampling units based on age group from household registries and economic status, sex, and geographical area [23]. All interviews and examinations were conducted in specially designed and equipped mobile centres that traveled throughout the country. All participants provided written informed consent to participate in the study, and the KNHANES studies were conducted according to the guidelines put forth in the Declaration of Helsinki. The study protocol was approved by the Institutional Review Board of the Korea Center for Disease Control and Prevention (IRB No: 2008-04EXP-01-C, 2009-01CON-032C, 2010-02CON-21-C). As the KNHANES data are deidentified and publicly available on the KNHANES website, this study was exempt from approval by the Institutional Review Board of the Kangbuk Samsung Hospital. We identified 1130 type 2 diabetic patients 19 years of age or older who received ophthalmic examinations. Diabetes mellitus was defined as diagnosis by a physician or the current use of blood glucose-lowering agents. After excluding patients with unavailable DR data $(n=243)$, a dataset of 887 patients was used in the final analysis.

\section{Data collection and definitions}

The KNHANES had four components: the Health Interview Survey, the Health Behavior Survey, the Health Examination Survey, and the Nutrition Survey. The health interviews, health behavior surveys, and health examinations were performed during a single day by trained medical staff and interviewers at mobile examination centres. One week after the health surveys, dieticians visited the homes of participants and conducted the nutrition survey [24]. Information on age, sex, health behaviors (smoking history and physical activity), history of physician-diagnosed disease (hypertension, diabetes, and dyslipidemia), and current medications was collected during the interview. Physical activity classification was based on the International Physical Activity Questionnaire short-form scoring protocol, and the participant's physical activity was classified as either "regular physical activity" when participants engaged in moderate-intensity activity more than five times per week or in vigorous activity more than three times per week or "other" [25]. A current smoker was defined as an individual who smoked cigarettes at the time of the interview.

Blood pressure (BP) was measured using a standard mercury sphygmomanometer (Baumanometer, W.A. Baum Co., Copiague, NY, USA) after the subject had rested for 5 min in a sitting position. Two systolic and diastolic BP readings were recorded at 5-min intervals and averaged for analysis.

Blood samples were obtained after a 12-h overnight fast, and levels of fasting plasma glucose (FPG), triglycerides (TG), and high-density lipoprotein cholesterol (HDL-C) were measured using a Hitachi 700-110 Chemistry Analyser (Hitachi, Tokyo, Japan). Glycosylated hemoglobin A1c (HbA1c) was measured using high-performance liquid chromatography (HLC-723G, Tosoh, Tokyo, Japan). An immunoradiometric assay using a 1470 WIZARD gammacounter (PerkinElmer, Finland) was used to measure serum insulin. Homeostasis model assessment of insulin resistance (HOMA-IR) was calculated using the following formula: HOMA-IR $=$ FPG $(\mathrm{mg} / \mathrm{dL}) \times$ fasting insulin $(\mu \mathrm{IU} / \mathrm{mL}) / 405$ [26]. We also calculated the homeostatic model assessment of $\beta$-cell function (HOMA- $\beta$ ) by using the established formula: HOMA- $\beta(\%)=[360 \times$ fasting insulin $(\mu \mathrm{IU} / \mathrm{mL})] /$ [FPG (mg/dL)-63] [26].

Other metabolic components, such as high BP and lipid abnormalities, were defined as a combination of a physician's diagnosis, the use of antihypertensive or lipidlowering medications, and the National Cholesterol Education Program Adult Treatment Panel III guidelines [systolic $\mathrm{BP} \geq 130 \mathrm{mmHg}$ and diastolic $\mathrm{BP} \geq 85 \mathrm{mmHg}$; TG level $\geq 150 \mathrm{mg} / \mathrm{dL}$; and low HDL-C level $(<40 \mathrm{mg} / \mathrm{dL}$ for men and $<50 \mathrm{mg} / \mathrm{dL}$ for women)] [27]. 


\section{Anthropometric measurements of obesity}

The body weight and height of subjects were measured with the participants wearing light clothing and no shoes, and BMI was calculated as weight $(\mathrm{kg})$ over height squared $\left(\mathrm{m}^{2}\right)$. Waist circumference was measured at the narrowest point between the lower border of the rib cage and the iliac crest. Obesity was defined as a BMI $\geq 25 \mathrm{~kg} / \mathrm{m}^{2}$ according to the criteria for the Asia-Pacific region [28].

The body fat mass and the percentage of body fat (fat mass $\times 100$ / total mass) were measured using whole-body DXA scans (QDR 4500 A fanbeam densitometer, Hologic Inc., Bedford, MA, USA) conducted by trained technicians in accordance with the manufacturer's procedures [22]. The DXA scans were performed with the participants wearing light clothing and no items that could interfere with the results. Data were analysed using Hologic Discovery software in its default configuration. Examinations that revealed items that could affect the accuracy of DXA results, such as prosthetic devices, implants, or other extraneous objects, were recorded as missing in the DXA dataset. The DXA instruments were calibrated according to the methods used in the NHANES surveys, and the reference values were obtained with the calibration method [29, 30]. DXA calibrations were maintained through an internal referencing system, which periodically measured bone and soft tissue equivalent reference standards during the examination.

\section{Assessment of diabetic retinopathy}

All participants underwent detailed ocular examinations, including measurement of visual acuity and intraocular pressure, autorefraction, slit lamp biomicroscopy, and fundus photography. Certified ophthalmologists performed all ocular examinations, and the Epidemiologic Survey Committee of the Korean Ophthalmologic Society verified the quality of the ophthalmic surveys [31]. Digital fundus photographs were taken with a non-mydriatic fundus camera (TRC-NW6S, Topcon, Tokyo, Japan; Nikon D-80, Nikon, Tokyo, Japan). If participants had a history of diabetes or random plasma glucose $\geq 200 \mathrm{mg} / \mathrm{dL}$ and/or suspicion of DR from non-mydriatic fundus photography, seven standard photographs from the Early Treatment for Diabetic Retinopathy Study protocol were obtained from each eye after pharmacological pupil dilation [32]. Diabetic retinopathy was diagnosed as the presence of one or more retinal microaneurysms or retinal blot hemorrhages with or without other lesions [33]. Clinically significant macular oedema (CSMO) was considered present when the macular oedema was within $500 \mathrm{~mm}$ of the foveal centre or if focal laser photocoagulation scars were present in the macular area. Participants were divided into three groups according to DR status: no DR, mild/moderate DR (hard exudates and cotton wool spots), and vision-threatening DR (intraretinal microvascular abnormalities, venous beading, new retinal vessels, vitreous or preretinal hemorrhage, fibrous proliferations, tractional retinal detachment, and CSMO) [34]. Data from the participant's most severe eye were used, and patients with ungradable fundus photographs were excluded from the analysis.

\section{Statistical analysis}

All analyses were performed using STATA SE 9.2 (STATA Corp., TX, USA). Descriptive statistics were presented as median (inter-quartile range) or percentage. Differences in the characteristics of participants according to DR status were evaluated using a Wilcoxon rank-sum test or $\chi^{2}$ test. Body mass index was assessed using both continuous and categorical variables as defined by the Asian criteria, and waist circumference and total body fat were analysed as continuous and categorical variables (by tertile). A multiple logistic regression model was used to evaluate the associations of BMI, waist circumference, and total body fat with the presence and severity of DR, adjusted for potential confounding factors that included age, sex, health-related habits (smoking, alcohol drinking, and physical activity), metabolic comorbidities (high blood pressure and lipid abnormalities), and diabetic indices (duration, HbAlc level, HOMA-IR, and HOMA- $\beta$ ). Odds ratios (ORs) and 95\% confidence intervals (CIs) were calculated. All statistical tests were two-tailed, and results with $P<0.05$ were considered statistically significant.

\section{Results}

A total of 887 patients (men, $48.6 \%$ ) with type 2 diabetes were included in the analysis. The median age was 64 years (range, 22-93 years); of these patients, 185 (20.9\%) had DR, 130 (14.7\%) had mild/moderate DR, and 55 (6.2\%) had vision-threatening DR. Table 1 shows the clinical and demographic characteristics of study participants according to the presence of DR. Compared to patients without DR, patients with DR had a longer duration of diabetes, higher HbA1c level and HOMA-IR, and lower HOMA- $\beta$ (all $P<$ $0.05)$. The proportion of patients who exercised regularly or had a higher BP was significantly greater in the no DR group, and patients with low HDL-C level were more likely to have DR (all $P<0.05$ ).

\section{Associations between obesity indices and diabetic retinopathy}

Patients without DR had higher BMI than those with DR, and this trend was similarly observed for waist 
Table 1 Characteristics of patients with type 2 diabetes by the presence of retinopathy

\begin{tabular}{|c|c|c|c|}
\hline Variable & No DR $(n=702)$ & $\mathrm{DR}(n=185)$ & $P$ value \\
\hline Age, median (IQR), years & $65(56-70)$ & $63(55-69)$ & 0.063 \\
\hline Men, $n(\%)$ & $353(50.3)$ & $78(42.2)$ & 0.049 \\
\hline \multicolumn{4}{|l|}{ Health-related habits } \\
\hline Current smoking, $n(\%)$ & $139(19.9)$ & $32(17.5)$ & 0.460 \\
\hline Problem drinking, $n(\%)$ & $54(7.7)$ & $7(3.8)$ & 0.062 \\
\hline Regular physical activity & $198(28.4)$ & 34 (18.6) & 0.007 \\
\hline \multicolumn{4}{|l|}{ Diabetes-related indices } \\
\hline Duration, median (IQR), years & $5(2-10)$ & $10(4-16)$ & $<0.001$ \\
\hline HbAlc, median (IQR), \% & $6.8(6.2-7.6)$ & $7.8(7.0-8.9)$ & $<0.001$ \\
\hline HOMA-IR, median (IQR) & $3.0(2.2-4.2)$ & $3.4(2.5-4.8)$ & 0.009 \\
\hline HOMA- $\beta$, median (IQR), \% & $55.7(38.5-80.0)$ & $43.4(21.6-74.5)$ & $<0.001$ \\
\hline \multicolumn{4}{|l|}{ Metabolic co-morbidities $^{\mathrm{a}}$} \\
\hline High blood pressure, $n(\%)$ & $509(72.5)$ & $113(61.1)$ & 0.003 \\
\hline Hypertriglyceridemia, $n(\%)$ & $418(59.5)$ & $120(64.9)$ & 0.188 \\
\hline Low HDL-C level, $n(\%)$ & $399(56.8)$ & $121(65.4)$ & 0.035 \\
\hline \multicolumn{4}{|l|}{ Obesity indices } \\
\hline BMI, median (IQR), $\mathrm{kg} / \mathrm{m}^{2}$ & $24.6(22.8-26.4)$ & $23.8(21.8-25.8)$ & 0.001 \\
\hline $\begin{array}{l}\text { Waist circumference, median } \\
\text { (IQR), cm }\end{array}$ & $87.0(81.6-93.0)$ & $85.4(79.8-90.6)$ & 0.024 \\
\hline Total body fat ${ }^{\mathrm{b}}$, median (IQR), \% & $29.4(23.2-35.3)$ & $27.9(22.1-33.6)$ & 0.102 \\
\hline
\end{tabular}

$B M I$ body mass index, $D R$ diabetic retinopathy, $H b A l c$ hemoglobin A1c, $H D L-C$ high-density lipoprotein cholesterol, HOMA homeostatic model assessment, $I Q R$ interquartile range, $I R$ insulin resistance

$P$ values were from Wilcoxon rank sum test or $\chi^{2}$ test

a'Defined as a combination of a physician's diagnosis, the use of antihypertensive or lipid-lowering medications, and the National Cholesterol Education Program Adult Panel III guidelines. High blood pressure (systolic blood pressure $\geq 130 \mathrm{mmHg}$, diastolic blood pressure $\geq 85 \mathrm{mmHg}$ ), hypertriglyceridemia $(\geq 150 \mathrm{mg} / \mathrm{dL}$ ), low HDL-C level $(<40 \mathrm{mg} / \mathrm{dL}$ for men and $<50 \mathrm{mg} / \mathrm{dL}$ for women)

${ }^{\mathrm{b}}$ Measured using a dual-energy X-ray absorptiometry

circumference $(P=0.001$ and $P=0.024$, respectively). Like BMI and waist circumference, total body fat was greater in the non-DR group than in the DR group, though the difference was not significant $(P=0.102$; Table 1$)$.

Multivariate analyses of obesity indices and the severity of DR indicated that BMI (adjusted OR [aOR], 0.80; 95\% CI, 0.70-0.92; $P=0.001$ ), waist circumference (aOR, 0.96; 95\% CI, 0.91-1.00; $P=0.047$ ), and total body fat (aOR, $0.86 ; 95 \% \mathrm{CI}, 0.79-0.92 ; P<0.001)$ were significantly associated with having vision-threatening DR after adjustment for confounding factors (Table 2). These inverse associations were also observed in the categorical analyses of BMI, waist circumference, and total body fat. In multivariate adjustments after categorization of BMI using the Asian criteria, obese patients were associated with lower risk of vision-threatening DR than normal or underweight patients (aOR, 0.65; 95\% CI, 0.43-0.99; $P$ for trend $=$ 0.029). When waist circumference was categorized into tertiles, patients in the highest tertile had significantly lower odds of vision-threatening DR compared with those in the lowest tertile (aOR, 0.54; 95\% CI, 0.32-0.91; $P$ for trend $=$ $0.009)$. In addition, categorical analysis of total body fat showed a significant trend between increasing body fat and lower risk of vision-threatening DR $(P$ for trend $=0.006)$, although each tertile of total body fat failed to reach statistical significance. However, for mild/moderate DR, protective effects of BMI, waist circumference, and total body fat were not observed by either continuous or categorical analyses (Table 2).

Sex difference in the association between body fat and diabetic retinopathy

Table 3 shows the associations between obesity indices and the presence of DR stratified by sex. In multivariate analyses, no significant associations between DR and BMI or waist circumference were found in either of the sexes with type 2 diabetes (all $P>0.05$ ). However, in women, total body fat was significantly associated with the presence of DR (aOR, 0.93; 95\% CI, 0.88-0.98; $P=0.009$; Table 3). After adjusting for BMI and waist circumference, there was a significant inverse relationship between total body fat and severity of DR in women $(P$ for trend $=0.004)$, but not in men $(P$ for trend $=0.126$; Fig. 1$)$.

\section{Discussion}

In this study, we evaluated the relationships between anthropometric parameters of obesity and the presence and severity of DR in a nationwide population-based survey in Korea. Our study showed that BMI, waist circumference, and total body fat were independently and inversely associated with the presence of vision-threatening DR after adjusting for covariates. Sex-stratified analyses revealed that total body fat was significantly associated with lower prevalence of DR in women with type 2 diabetes. Our results suggest that a high fat composition might play a protective role in the development and progression of DR in women with type 2 diabetes.

Many epidemiologic studies have explored the association of DR with BMI or anthropometric parameters, but the conclusions have been contradictory. Recent studies conducted in Asian populations have reported an inverse association between BMI and the risk of DR, suggesting a protective role for higher BMI in the development of DR. For instance, the Singapore Epidemiology of Eye Diseases cohort studies found evidence of an inverse association of BMI with the presence and severity of DR, and the Shanghai Diabetes Registry Database study, which included 2533 patients with type 2 diabetes, also revealed that overweight patients have lower DR risk than normal weight subjects [19, 35, 36]. However, the studies from Western countries have reported contradictory results: the Hoorn study in the Netherlands found an adverse effect of a higher BMI on DR, and the Diabetes Management Project study conducted in Australia similarly demonstrated a significant association between higher BMI and the risk of DR $[6,14]$. 
Table 2 Associations between obesity indices and diabetic retinopathy (reference to 702 patients without retinopathy)

\begin{tabular}{|c|c|c|c|c|c|c|}
\hline \multirow[t]{2}{*}{ Obesity indices } & \multicolumn{2}{|l|}{ Any DR $(n=185)$} & \multicolumn{2}{|c|}{ Mild/moderate DR $(n=130)$} & \multicolumn{2}{|c|}{ Vision-threatening DR $(n=55)$} \\
\hline & $O R^{\mathrm{a}}(95 \% \mathrm{CI})$ & $P$ value & $\mathrm{OR}^{\mathrm{a}}(95 \% \mathrm{CI})$ & $P$ value & $\mathrm{OR}^{\mathrm{a}}(95 \% \mathrm{CI})$ & $P$ value \\
\hline BMI (per $1 \mathrm{~kg} / \mathrm{m}^{2}$ ) & $0.94(0.88-1.01)$ & 0.075 & $0.98(0.92-1.06)$ & 0.682 & $0.80(0.70-0.92)$ & 0.001 \\
\hline$<23.0$ & 1 & & 1 & & 1 & \\
\hline $23-24.9$ & $0.77(0.46-1.29)$ & & $1.01(0.57-1.79)$ & & $0.37(0.14-0.97)$ & \\
\hline$\geq 25.0$ & $0.90(0.71-1.14)$ & & $1.01(0.77-1.32)$ & & $0.65(0.43-0.99)$ & \\
\hline$P$ for trend & & 0.219 & & 0.809 & & 0.029 \\
\hline Waist circumference (per $1 \mathrm{~cm}$ ) & $0.99(0.97-1.01)$ & 0.362 & $1.00(0.98-1.02)$ & 0.987 & $0.96(0.91-1.00)$ & 0.047 \\
\hline Tertile $1(<83)$ & 1 & & 1 & & 1 & \\
\hline Tertile $2(83-90)$ & $0.83(0.51-1.33)$ & & $0.97(0.57-1.66)$ & & $0.54(0.24-1.23)$ & \\
\hline Tertile $3(>90)$ & $0.78(0.60-1.02)$ & & $0.88(0.66-1.18)$ & & $0.54(0.32-0.91)$ & \\
\hline$P$ for trend & & 0.044 & & 0.281 & & 0.009 \\
\hline Total body fat (per 1\%) & $0.94(0.90-0.98)$ & 0.002 & $0.97(0.92-1.01)$ & 0.164 & $0.86(0.79-0.92)$ & $<0.001$ \\
\hline Tertile $1(<25)$ & 1 & & 1 & & 1 & \\
\hline Tertile $2(25-33)$ & $0.66(0.36-1.19)$ & & $0.91(0.46-1.80)$ & & $0.38(0.13-1.05)$ & \\
\hline Tertile 3 (>33) & $0.69(0.41-1.16)$ & & $0.81(0.45-1.49)$ & & $0.48(0.22-1.06)$ & \\
\hline$P$ for trend & & 0.024 & & 0.206 & & 0.006 \\
\hline
\end{tabular}

$B M I$ body mass index, $C I$ confidence interval, $D R$ diabetic retinopathy, $O R$ odds ratio

${ }^{a}$ Adjusted for age, sex, health-related habits (smoking, alcohol drinking, and physical activity), metabolic comorbidities (high blood pressure and lipid abnormalities), and diabetic indices (duration, hemoglobin A1c level, HOMA-IR, and HOMA- $\beta$ )

Table 3 Sex-stratified analysis between obesity indices and diabetic retinopathy

\begin{tabular}{|c|c|c|c|c|c|c|}
\hline \multirow[t]{2}{*}{ Obesity indices } & \multirow[t]{2}{*}{ Range } & \multicolumn{2}{|l|}{ Men } & \multirow[t]{2}{*}{ Range } & \multicolumn{2}{|l|}{ Women } \\
\hline & & No. of patients & $\mathrm{OR}^{\mathrm{a}}(95 \% \mathrm{CI})$ & & No. of patients & $\mathrm{OR}^{\mathrm{a}}(95 \% \mathrm{CI})$ \\
\hline BMI $\left(\right.$ per $\left.1 \mathrm{~kg} / \mathrm{m}^{2}\right)$ & & & $0.97(0.87-1.08)$ & & & $0.92(0.84-1.01)$ \\
\hline Normal & $<23.0$ & 144 & 1 & $<23.0$ & 129 & 1 \\
\hline Overweight & $23-24.9$ & 118 & $0.52(0.24-1.12)$ & $23-24.9$ & 103 & $1.12(0.53-2.32)$ \\
\hline Obesity & $\geq 25.0$ & 169 & $0.86(0.60-1.24)$ & $\geq 25.0$ & 224 & $0.96(0.69-1.34)$ \\
\hline$P$ for trend & & & 0.327 & & & 0.516 \\
\hline Waist circumference (per $1 \mathrm{~cm}$ ) & & & $0.99(0.96-1.02)$ & & & $0.99(0.96-1.02)$ \\
\hline Tertile 1 & $<84$ & 134 & 1 & $<82$ & 140 & 1 \\
\hline Tertile 2 & $84-90$ & 140 & $0.80(0.39-1.67)$ & $82-89$ & 151 & $0.57(0.28-1.15)$ \\
\hline Tertile 3 & $>90$ & 157 & $0.72(0.49-1.06)$ & $>89$ & 165 & $0.86(0.60-1.22)$ \\
\hline$P$ for trend & & & 0.076 & & & 0.270 \\
\hline Body fat (per $1 \%$ ) & & & $0.94(0.89-1.01)$ & & & $0.93(0.88-0.98)$ \\
\hline Tertile 1 & $<20$ & 119 & 1 & $<32$ & 145 & 1 \\
\hline Tertile 2 & $20-25$ & 159 & $0.98(0.47-2.05)$ & $32-36$ & 132 & $0.62(0.33-1.18)$ \\
\hline Tertile 3 & $>25$ & 153 & $0.80(0.53-1.23)$ & $>36$ & 179 & $0.76(0.54-1.07)$ \\
\hline$P$ for trend & & & 0.201 & & & 0.028 \\
\hline
\end{tabular}

$B M I$ body mass index, $C I$ confidence interval, $D R$ diabetic retinopathy, $O R$ odds ratio

${ }^{a}$ Adjusted for age, health-related habits (smoking, alcohol drinking, and physical activity), metabolic comorbidities (high blood pressure and lipid abnormalities), and diabetic indices (duration, hemoglobin A1c level, HOMA-IR, and HOMA- $\beta$ )

This discrepancy might be explained partly by differences in study methodology as well as by ethnic differences in study participants. Previous reports have shown that the BMIs of Caucasian diabetics were much higher than the BMIs of Asian diabetics; and that Caucasian diabetic patients have higher BMIs compared to the BMIs of Caucasian non-diabetic subjects, while Asian diabetic patients have similar BMIs compared to the BMIs of Asian nondiabetic subjects [37, 38]. These differences reflect the difficulties in comparing BMI data of diabetic patients from 


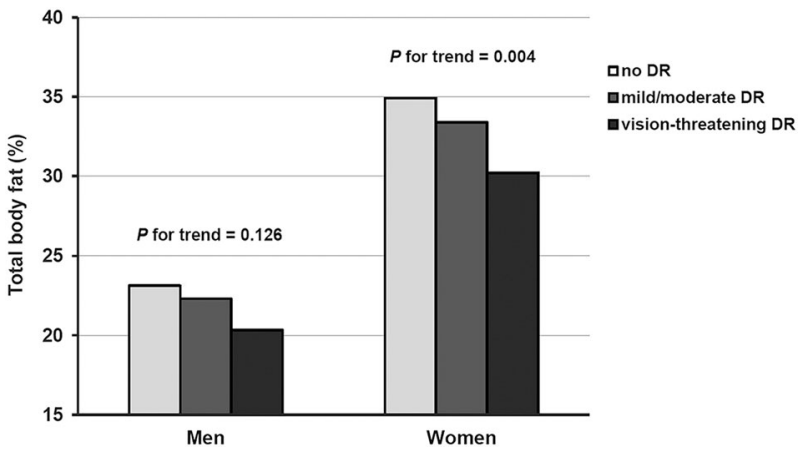

Fig. 1 Associations between total body fat and the severity of diabetic retinopathy stratified by sex. DR indicates diabetic retinopathy. $P$ values were from multivariate regression model adjusted for all variables in Table 3 plus body mass index and waist circumference

different ethnic groups. In general, obese diabetic patients have greater insulin secretion, but show more insulin resistance than non-obese diabetics [39]. Furthermore, patients with a higher BMI were reported to require less insulin treatments, suggesting good beta cell function, and this might support a protective role of obesity in DR [18].

Obesity is a risk factor for diabetes and possibly for diabetes-associated microvascular complications, which include retinopathy. While BMI is the most frequently used indicator of obesity, results from epidemiologic studies supporting the association between obesity, as represented by BMI, and DR have been inconsistent, and increasing evidence suggests that an obesity index should account for abdominal obesity and body fat distribution when evaluating the effect of obesity on DR. In general, higher BMI indicates overall obesity, whereas abdominal obesity is assessed by waist circumference or waist-hip ratio. However, these anthropometric measurements can show a variable correlation according to metabolic disorder and an inaccurate status of obesity because they do not distinguish body fat from other components. Therefore, the measurement of body fat using DXA, computerized tomography (CT), or magnetic resonance imaging (MRI) can provide more accurate information on obesity. In this study, total body fat was measured with DXA, and we found that total body fat, independent of BMI and waist circumference, was significantly associated with lower risk of vision-threatening DR, and that women with greater body fat might be less likely to have DR.

The mechanism underlying the effect of total body fat on DR is unclear. A possible explanation for the association of adiposity with low risk of DR is adipokine production, especially adiponectin that is expressed mostly in white adipose tissues [40]. Adiponectin plays a key role in metabolic modulations involved in neovascular eye disorders, such as retinopathy of prematurity, diabetic retinopathy, and age-related macular degeneration [41-44]. In fact, dyslipidemia, as well as hyperglycemia, together with induced oxidative stress are well-known risk factors for the development of DR [45]. In patients with type 2 diabetes, adiponectin appears to modulate lipid metabolism, leading to the reduction of cholesterol, and to increase peripheral insulin sensitivity by controlling glycogen synthesis [46, 47]. Furthermore, a previous study has reported that retinal blood flow in patients with early DR was positively correlated with the level of serum adiponectin [43]. However, the results from studies on the association between serum adiponectin level and DR in type 2 diabetic patients are controversial and should be interpreted cautiously. Some reports have shown that high adiponectin level is closely correlated with severe DR, especially in proliferative retinopathy, whereas others suggest a protective role of adiponectin in the development and progression of DR in type 2 diabetic patients [42, 48, 49]. Elevated serum adiponectin level might be compensatory and beneficial in diabetic patients with proliferative retinopathy. Further prospective studies are needed to investigate the causative relationship between adiponectin and DR.

Previous epidemiologic studies have reported that abdominal obesity assessed by waist-hip ratio was significantly associated with DR prevalence in women only, while general obesity, as defined by BMI, was shown to be inversely associated with DR prevalence and severity regardless of sex $[18,20]$. In this study both general and abdominal obesity, as assessed by BMI or waist circumference, respectively, were inversely associated with the presence of DR, especially vision-threatening retinopathy. Direct comparisons with previous findings, however, could not be allowed due to differences in study participants and methodology, and the biological mechanism of the association between abdominal obesity and DR is still not clearly understood.

In the sex-stratified multivariate analysis, the protective associations between obesity indices and the presence of DR were observed for total body fat in women, whereas this association was not significant in men. The sex-specific effect of body fat on DR might be related to intrinsic hormonal responses or body fat distribution. Some studies have suggested that estrogen might play an important role in body fat distribution in women, who have more peripheral and lower body fat deposition, than in men, who typically have more truncal and upper body fat deposition [50, 51]. On the other hand, a relatively narrow range of total body fat in men in our study population might be a possible reason for the lack of association between body fat and DR. Overall, the mechanisms of the sex-specific association between body fat and DR remain unknown, and further studies including longitudinal and experimental research are warranted to investigate the underlying mechanism.

The strengths of this study include the participants that are representative of the nationwide population of Korea, the large sample size, and the use of standardized fundus grading protocols with seven standard photographs. Furthermore, 
unlike previous studies, total body fat assessed by DXA was used together with BMI and waist circumference for the evaluation of obesity-related DR prevalence and progression. However, this study has some limitations. First, the crosssectional nature of the design does not allow cause-effect relationships to be concluded. Second, socioeconomic status, which might act as a confounding factor in the evaluation of the anthropometric parameters of obesity, was not considered in this study. Third, although abdominal fat mass is a good indicator of abdominal obesity and a well-known risk factor for diabetes, we could not assess the association between abdominal fat and DR because the KNHANES data do not include results such as CT or MRI images for participants. Fourth, due to the exclusion of participants with type 1 diabetes, the association between DR and BMI or body fat in type 1 diabetic patients could not be investigated. Further studies are needed to determine the effect of adiposity on DR in patients with type 1 diabetes.

In summary, our study uncovers an independent association of body fat with DR and provides evidence that sex differences in body fat composition may affect the prevalence and progression of DR. Longitudinal studies collaborating experimental research are warranted to confirm and further characterize our findings.

\section{Summary}

\section{What was known before}

- Obesity is presumably a risk factor for diabetic microvascular complications including retinopathy. There have been conflicting reports of the relationship between obesity and diabetic retinopathy.

\section{What this study adds}

- In Korean patients with type 2 diabetes, higher body mass index, larger waist circumference, and higher total body fat were inversely associated with the risk of vision-threatening retinopathy. Women with greater body fat showed less prevalence and progression of diabetic retinopathy.

Acknowledgements We thank all members in the Epidemiologic Survey Committee of the Korean Ophthalmologic Society for conducting ophthalmologic examinations in the KNHANES and for supplying data.

\section{Compliance with ethical standards}

Conflict of interest The authors declare that they have no conflict of interest.
Publisher's note: Springer Nature remains neutral with regard to jurisdictional claims in published maps and institutional affiliations.

\section{References}

1. Guariguata L, Whiting DR, Hambleton I, Beagley J, Linnenkamp U, Shaw JE. Global estimates of diabetes prevalence for 2013 and projections for 2035. Diabetes Res Clin Pract. 2014;103:137-49.

2. Antonetti DA, Klein R, Gardner TW. Diabetic retinopathy. N Engl J Med. 2012;366:1227-39.

3. Wong TY, Cheung N, Tay WT, Wang JJ, Aung T, Saw SM, et al. Prevalence and risk factors for diabetic retinopathy: the Singapore Malay Eye Study. Ophthalmology. 2008;115:1869-75.

4. Trento M, Passera P, Trevisan M, Schellino F, Sitia E, Albani S, et al. Quality of life, impaired vision and social role in people with diabetes: a multicenter observational study. Acta Diabetol. 2013;50:873-7.

5. Yau JW, Rogers SL, Kawasaki R, Lamoureux EL, Kowalski JW, Bek T, et al. Global prevalence and major risk factors of diabetic retinopathy. Diabetes Care. 2012;35:556-64.

6. van Leiden HA, Dekker JM, Moll AC, Nijpels G, Heine RJ, Bouter LM, et al. Blood pressure, lipids, and obesity are associated with retinopathy: the hoorn study. Diabetes Care. 2002;25:1320-5.

7. Brown JB, Pedula KL, Summers KH. Diabetic retinopathy: contemporary prevalence in a well-controlled population. Diabetes Care. 2003;26:2637-42.

8. Beulens JW, Patel A, Vingerling JR, Cruickshank JK, Hughes $\mathrm{AD}$, Stanton A, et al. Effects of blood pressure lowering and intensive glucose control on the incidence and progression of retinopathy in patients with type 2 diabetes mellitus: a randomised controlled trial. Diabetologia. 2009;52:2027-36.

9. Ismail-Beigi F, Craven T, Banerji MA, Basile J, Calles J, Cohen $\mathrm{RM}$, et al. Effect of intensive treatment of hyperglycaemia on microvascular outcomes in type 2 diabetes: an analysis of the ACCORD randomised trial. Lancet. 2010;376:419-30.

10. Garvey WT, Garber AJ, Mechanick JI, Bray GA, Dagogo-Jack S, Einhorn D, et al. American association of clinical endocrinologists and american college of endocrinology position statement on the 2014 advanced framework for a new diagnosis of obesity as a chronic disease. Endocr Pract. 2014;20:977-89.

11. Khalangot M, Tronko M, Kravchenko V, Kulchinska J, Hu G. Body mass index and the risk of total and cardiovascular mortality among patients with type 2 diabetes: a large prospective study in Ukraine. Heart. 2009;95:454-60.

12. Eeg-Olofsson K, Cederholm J, Nilsson PM, Zethelius B, Nunez L, Gudbjörnsdóttir S, et al. Risk of cardiovascular disease and mortality in overweight and obese patients with type 2 diabetes: an observational study in 13,087 patients. Diabetologia. 2009;52:65-73.

13. Maric C, Hall JE. Obesity, metabolic syndrome and diabetic nephropathy. Contrib Nephrol. 2011;170:28-35.

14. Dirani M, Xie J, Fenwick E, Benarous R, Rees G, Wong TY, et al. Are obesity and anthropometry risk factors for diabetic retinopathy? The diabetes management project. Invest Ophthalmol Vis Sci. 2011;52:4416-21.

15. Chaturvedi N, Fuller JH. Mortality risk by body weight and weight change in people with NIDDM. The WHO Multinational Study of Vascular Disease in Diabetes. Diabetes Care. 1995; 18:766-74.

16. Henricsson M, Nyström L, Blohmé G, Ostman J, Kullberg C, Svensson M, et al. The incidence of retinopathy 10 years after diagnosis in young adult people with diabetes: results from the 
nationwide population-based Diabetes Incidence Study in Sweden (DISS). Diabetes Care. 2003;26:349-54.

17. Klein R, Klein BE, Moss SE. Is obesity related to microvascular and macrovascular complications in diabetes? The Wisconsin Epidemiologic Study of Diabetic Retinopathy. Arch Intern Med. 1997;157:650-6.

18. Raman R, Rani PK, Gnanamoorthy P, Sudhir RR, Kumaramanikavel G, Sharma T. Association of obesity with diabetic retinopathy: Sankara Nethralaya Diabetic Retinopathy Epidemiology and Molecular Genetics Study (SN-DREAMS Report no. 8). Acta Diabetol. 2010;47:209-15.

19. Lu J, Hou X, Zhang L, Jiang F, Hu C, Bao Y, et al. Association between body mass index and diabetic retinopathy in Chinese patients with type 2 diabetes. Acta Diabetol. 2015;52:701-8.

20. Man RE, Sabanayagam C, Chiang PP, Li LJ, Noonan JE, Wang $\mathrm{JJ}$, et al. Differential association of generalized and abdominal obesity with diabetic retinopathy in Asian patients with type 2 diabetes. JAMA Ophthalmol. 2016;134:251-7.

21. Lee CM, Huxley RR, Wildman RP, Woodward M. Indices of abdominal obesity are better discriminators of cardiovascular risk factors than BMI: a meta-analysis. J Clin Epidemiol. 2008;61:646-53.

22. Pietrobelli A, Formica C, Wang Z, Heymsfield SB. Dual-energy X-ray absorptiometry body composition model: review of physical concepts. Am J Physiol. 1996;271:E941-951.

23. Choi YJ, Kim HC, Kim HM, Park SW, Kim J, Kim DJ. Prevalence and management of diabetes in Korean adults: Korea National Health and Nutrition Examination Surveys 1998-2005. Diabetes Care. 2009;32:2016-20.

24. Kweon S, Kim Y, Jang MJ, Kim Y, Kim K, Choi S, et al. Data resource profile: the Korea National Health and Nutrition Examination Survey (KNHANES). Int J Epidemiol. 2014; 43:69-77.

25. Craig CL, Marshall AL, Sjöström M, Bauman AE, Booth ML, Ainsworth BE, et al. International physical activity questionnaire: 12-country reliability and validity. Med Sci Sports Exerc. 2003;35:1381-95.

26. Matthews DR, Hosker JP, Rudenski AS, Naylor BA, Treacher DF, Turner RC. Homeostasis model assessment: insulin resistance and beta-cell function from fasting plasma glucose and insulin concentrations in man. Diabetologia. 1985;28:412-9.

27. Lee SY, Park HS, Kim DJ, Han JH, Kim SM, Cho GJ, et al. Appropriate waist circumference cutoff points for central obesity in Korean adults. Diabetes Res Clin Pract. 2007;75:72-80.

28. Oh SW. Obesity and metabolic syndrome in Korea. Diabetes Metab J. 2011:35:561-6.

29. Kelly TL, Wilson KE, Heymsfield SB. Dual energy X-ray absorptiometry body composition reference values from NHANES. PLoS ONE. 2009;4:e7038.

30. Schoeller DA, Tylavsky FA, Baer DJ, Chumlea WC, Earthman CP, Fuerst T, et al. QDR 4500A dual-energy X-ray absorptiometer underestimates fat mass in comparison with criterion methods in adults. Am J Clin Nutr. 2005;81:1018-25.

31. Yoon KC, Mun GH, Kim SD, Kim SH, Kim CY, Park KH, et al. Prevalence of eye diseases in South Korea: data from the Korea National Health and Nutrition Examination Survey 2008-2009. Korean J Ophthalmol. 2011;25:421-33.

32. Grading diabetic retinopathy from stereoscopic color fundus photographs-an extension of the modified Airlie House classification. ETDRS report number 10. Early Treatment Diabetic Retinopathy Study Research Group. Ophthalmology. 1991;98:786-806.

33. Klein R, Klein BE, Moss SE, Cruickshanks KJ. The Wisconsin Epidemiologic Study of Diabetic Retinopathy: XVII. The 14-year incidence and progression of diabetic retinopathy and associated risk factors in type 1 diabetes. Ophthalmology. 1998;105:180115.

34. Kempen JH, O'Colmain BJ, Leske MC, Haffner SM, Klein R, Moss SE, et al. The prevalence of diabetic retinopathy among adults in the United States. Arch Ophthalmol. 2004; 122:552-63.

35. Lim LS, Tai ES, Mitchell P, Wang JJ, Tay WT, Lamoureux E, et al. C-reactive protein, body mass index, and diabetic retinopathy. Invest Ophthalmol Vis Sci. 2010;51:4458-63.

36. Rooney D, Lye WK, Tan G, Lamoureux EL, Ikram MK, Cheng $\mathrm{CY}$, et al. Body mass index and retinopathy in Asian populations with diabetes mellitus. Acta Diabetol. 2015;52:73-80.

37. Deurenberg P, Yap M, van Staveren WA. Body mass index and percent body fat: a meta analysis among different ethnic groups. Int J Obes Relat Metab Disord. 1998;22:1164-71.

38. Sone H, Ito H, Ohashi Y, Akanuma Y, Yamada N, Japan Diabetes Complication Study Group. Obesity and type 2 diabetes in Japanese patients. Lancet. 2003;361:85.

39. Jensen CC, Cnop M, Hull RL, Fujimoto WY, Kahn SE, American Diabetes Association GENNID Study Group. Beta-cell function is a major contributor to oral glucose tolerance in high-risk relatives of four ethnic groups in the US. Diabetes. 2002;51:2170-8.

40. Joyal JS, Sun Y, Gantner ML, Shao Z, Evans LP, Saba N, et al. Retinal lipid and glucose metabolism dictates angiogenesis through the lipid sensor Ffar1. Nat Med. 2016;22:439-45.

41. Fu Z, Lofqvist CA, Shao Z, Sun Y, Joyal JS, Hurst CG, et al. Dietary omega-3 polyunsaturated fatty acids decrease retinal neovascularization by adipose-endoplasmic reticulum stress reduction to increase adiponectin. Am J Clin Nutr. 2015;101:87988.

42. Mao D, Peng H, Li Q, Wang J, Li P, Hu K, et al. Aqueous humor and plasma adiponectin levels in proliferative diabetic retinopathy patients. Curr Eye Res. 2012;37:803-8.

43. Omae T, Nagaoka T, Yoshida A. Relationship between retinal blood flow and serum adiponectin concentrations in patients with type 2 diabetes mellitus. Invest Ophthalmol Vis Sci. 2015;56:4143-9.

44. Kaarniranta K, Paananen J, Nevalainen T, Sorri I, Seitsonen S, Immonen I, et al. Adiponectin receptor 1 gene (ADIPOR1) variant is associated with advanced age-related macular degeneration in Finnish population. Neurosci Lett. 2012;513:233-7.

45. Chang YC, Wu WC. Dyslipidemia and diabetic retinopathy. Rev Diabet Stud. 2013;10:121-32.

46. Wang M, Wang D, Zhang Y, Wang X, Liu Y, Xia M. Adiponectin increases macrophages cholesterol efflux and suppresses foam cell formation in patients with type 2 diabetes mellitus. Atherosclerosis. 2013;229:62-70.

47. Yokoyama H, Emoto M, Mori K, Araki T, Teramura M, Koyama $\mathrm{H}$, et al. Plasma adiponectin level is associated with insulinstimulated nonoxidative glucose disposal. J Clin Endocrinol Metab. 2006;91:290-4.

48. Jung CH, Kim BY, Mok JO, Kang SK, Kim CH. Association between serum adipocytokine levels and microangiopathies in patients with type 2 diabetes mellitus. J Diabetes Investig. 2014;5:333-9.

49. Fu Z, Gong Y, Löfqvist C, Hellström A, Smith LE. Review: adiponectin in retinopathy. Biochim Biophys Acta. 2016;1862:1392-1400.

50. Shimokata H, Tobin JD, Muller DC, Elahi D, Coon PJ, Andres R. Studies in the distribution of body fat: I. Effects of age, sex, and obesity. J Gerontol. 1989;44:M66-73.

51. Cascio C, Deidda I, Russo D, Guarneri P. The estrogenic retina: The potential contribution to healthy aging and age-related neurodegenerative diseases of the retina. Steroids. 2015;103:31-41. 